07

\title{
Прецизионный алгоритм переключения мемристора в состояние с заданным сопротивлением
}

\author{
(C) К.Э. Никируй ${ }^{1,2}$, А.В. Емельянов ${ }^{1,2}$, В.А. Демин ${ }^{1,2}$, \\ В.В. Рыльков ${ }^{1}$, А.В. Ситников ${ }^{3}$, П.К. Кашкаров ${ }^{1,2,4}$ \\ ${ }^{1}$ НИЦ „Курчатовский институт“, Москва, Россия \\ ${ }^{2}$ Московский фризико-технический институт (Государственный \\ университет), Долгопрудный, Московская обл., Россия \\ ${ }^{3}$ Воронежский государственный технический университет, \\ Воронеж, Россия \\ ${ }^{4}$ Московский государственный университет им. М.В. Ломоносова, \\ Москва, Россия \\ E-mail: NikiruyKristina@gmail.com
}

Поступило в Редакцию 30 октября 2017 г.

Развит алгоритм перевода мемристора в состояние с заданным сопротивлением с высокой точностью. Алгоритм основан на подаче импульсов напряжения с плавно растущей амплитудой и случайным образом изменяющейся длительностью в заданных пределах. Продемонстрирована возможность реализации алгоритма на примере мемристорных структур на основе нанокомпозита $\left(\mathrm{Co}_{40} \mathrm{Fe}_{40} \mathrm{~B}_{20}\right)_{x}\left(\mathrm{LiNbO}_{3}\right)_{100-x} \mathrm{c} x \approx 10$ at.\%. Подобраны оптимальные параметры для работы алгоритма с минимальным количеством итераций, позволившие достичь в этом случае точности задания сопротивления не хуже $0.5 \%$. Полученные результаты могут быть использованы при создании нейроморфных систем.

DOI: 10.21883/PJTF.2018.10.46095.17099

Мемристор - элемент электрической цепи, способный изменять свое сопротивление в зависимости от величины электрического поля и протекшего заряда [1]. Интерес к изучению мемристоров обусловлен перспективами их применения для создания энергонезависимой памяти с произвольным доступом (RRAM), обладающей низким энергопотреблением при записи информации $\left(\sim 10^{-16} \mathrm{~J} / \mathrm{bit}\right)[2,3]$, и нейроморфных систем [4-6]. При этом возможность задавать различные промежуточные состояния мемристора является необходимым условием для его ис- 
пользования в качестве аналога синапса в нейроморфных приложениях (распознавание образов и естественного языка, способности к обучению, обобщениям и пр.). Для автоматизации работы даже простейших нейроморфных систем необходимо разработать алгоритм переключения мемристора в состояние с заданным сопротивлением. В литературе предложено несколько вариантов алгоритмов переключения. Один из них заключается в использовании дополнительных элементов цепи [7], в том числе резисторов [8,9]. Резистор в данном случае выступает в качестве делителя напряжения. На последовательно соединенные резистор и мемристор в низкопроводящем состоянии подается постоянное напряжение, и по мере уменьшения сопротивления мемристора уменьшается падение напряжения на нем. Такой тип переключения позволяет увеличить устойчивость к переключениям (endurance) [10]. Однако существенными недостатками данного алгоритма являются косвенное управление итоговым сопротивлением мемристора и использование дополнительных элементов цепи, что повышает энергопотребление.

Другой вариант переключения мемристора в состояние с заданным сопротивлением основан на подаче импульсов напряжения с плавно растущей амплитудой [11]. В этом методе текущее сопротивление мемристора приближают к заданному значению, увеличивая амплитуду подаваемых импульсов постоянной длительности на небольшое значение. Знак амплитуды импульса определяется знаком разности между текущим сопротивлением и заданным. Данный алгоритм удобен простотой реализации. Однако этот алгоритм не является универсальным и в некоторых случаях неприменим, особенно когда необходима высокая точность задания сопротивления (см. далее). Поэтому цель настоящей работы состоит в разработке и реализации алгоритма переключения мемристора в состояние с заданным сопротивлением с высокой точностью.

Исследовались конденсаторного типа мемристорные структуры металл/нанокомпозит/металл $(M / \mathrm{HK} / M)$ на основе нанокомпозита $(\mathrm{HK})\left(\mathrm{Co}_{40} \mathrm{Fe}_{40} \mathrm{~B}_{20}\right)_{x}\left(\mathrm{LiNbO}_{3}\right)_{100-x}$ с содержанием сплава $\mathrm{Co}_{40} \mathrm{Fe}_{40} \mathrm{~B}_{20}$ $x \approx 10$ at.\%. Слои НК толщиной около $3 \mu \mathrm{m}$ были синтезированы методом ионно-лучевого распыления составной мишени при комнатной температуре и представляли собой ансамбль сильно вытянутых (до $10-15 \mathrm{~nm}$ ) в направлении роста НК CoFeB гранул с поперечным размером 2-4 nm [12,13]. Элементный состав НК определялся с использованием энергодисперсионной рентгеновской приставки Oxford

Письма в ЖТФ, 2018, том 44, вып. 10 


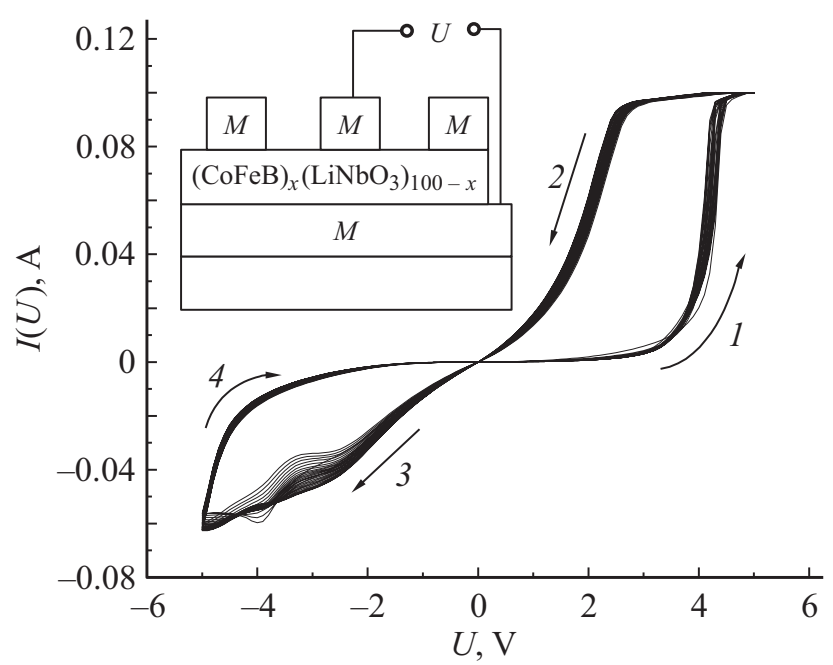

Рис. 1. ВАХ мемристорной структуры $M /(\mathrm{CoFeB})_{x}\left(\mathrm{LiNbO}_{3}\right)_{100-x} / M$ с содержанием сплава $\mathrm{CoFeB} x \approx 10$ at.\%. На вставке представлена схема исследованной структуры.

INCA Energy 250 на сканирующем электронном микроскопе JEOL JSM-6380 LV. Рабочий диапазон сопротивлений мемристора составлял $\sim 100-15000 \Omega$. Исследования резистивного переключения (РП) и вольт-амперных характеристик (BAX) мемристоров осуществлялись с помощью зондовой аналитической станции Cascade Microtech PM5. Импульсы напряжения подавались посредством источника-измерителя National Instruments PXIe-4139, программируемого в среде LABView.

На рис. 1 представлена типичная ВАХ мемристорной структуры $M /(\mathrm{CoFeB})_{x}\left(\mathrm{LiNbO}_{3}\right)_{100-x} / M$ c $x \approx 10$ at.\%. При приложении положительного напряжения мемристор переходит в низкоомное состояние, при приложении отрицательного - в высокоомное. Участки 1 и 4 ВАХ соответствуют высокоомному состоянию мемристора, 2 и 3 - низкоомному; отношение сопротивлений в этих состояниях $R_{o f f} / R_{o n} \approx 150$. Такой вид зависимости ВАX можно объяснить в рамках модели, предложенной в работе [13]. Согласно этой модели, РП структуры обусловлено несколькими факторами: 1) перколяционным характером 
проводимости НК и формированием при относительно небольших содержаниях металла изолированных цепочек из вытянутых гранул, которыми определяется поперечная проводимость структуры; 2) аномально сильным уменьшением сопротивления цепочек в полях $E>10^{4} \mathrm{~V} / \mathrm{cm}$ вследствие подавления эффектов кулоновской блокады [14] и генерации кислородных вакансий $\left.V_{\mathrm{O}}[15] ; 3\right)$ инжекцией вакансий $V_{\mathrm{O}}$ при напряжении на структуре $U>0$ в высокоомную сильно окисленную прослойку, формируемую преимущественно у нижнего электрода структуры $M / \mathrm{HK} / M$ в силу особенностей ее роста; 4) шунтированием прослойки и резистивным переключением структуры в низкоомное состояние (участки 2,3 на рис. 1 ); 5) экстракцией вакансий $V_{O}$ при $U<0$ из приконтактной прослойки, которая приводит к обратному переключению структуры в высокоомное состояние (участки 1,4 на рис. 1).

В рамках предложенной модели РП способность принимать произвольное резистивное состояние структуры $M / \mathrm{HK} / M$ в диапазоне между $R_{\text {on }}$ и $R_{\text {off }}$ объясняется возможностью частичного шунтирования приконтактной прослойки структуры при относительно небольшой длительности поданного импульса напряжения и/или его амплитуде $U$.

Предлагаемый в работе алгоритм переключения мемристора в состояние с заданным сопротивлением основан на подаче импульсов напряжения с плавно растущей амплитудой и в отличие от [11] со случайным образом изменяющейся длительностью. Основными входными параметрами алгоритма являются амплитуда первого импульса $U_{0}$, изменение амплитуды на следующем шаге $\Delta U$, сопротивление $R$, которое должен иметь мемристор, точность задания сопротивления $\Delta R / R$ и длительность обучающих импульсов $\tau$. Суть алгоритма состоит в следующем. Каждая итерация алгоритма делится на два этапа: измерение и обучение. На каждом этапе измерения подается импульс постоянной полярности, амплитуды и длительности $(+0.1 \mathrm{~V}, 50 \mathrm{~ms})$, измеряется ток и вычисляется текущее сопротивление $R_{i}$ на $i$-й итерации. На этапе обучения подаются импульсы с амплитудой $U_{i}$ и длительностью $\tau$. Амплитуда первого обучающего импульса всегда $U_{0}$, полярность всех импульсов выбирается такой же, как знак $\left(R_{i}-R\right)$. Далее, если знаки $\left(R-R_{i-1}\right)$ и $\left(R-R_{i}\right)$ одинаковы, амплитуда $i$-го обучающего импульса $U_{i}=U_{i-1}+\Delta U$; если различны, амплитуда $U_{i}=U_{0}$ и при этом изменяется полярность $i$-го импульса. Если на $i$-м шаге величина амплитуды $U_{i}$ превышает максимальное допустимое значение, амплитуда импульса

Письма в ЖТФ, 2018, том 44, вып. 10 


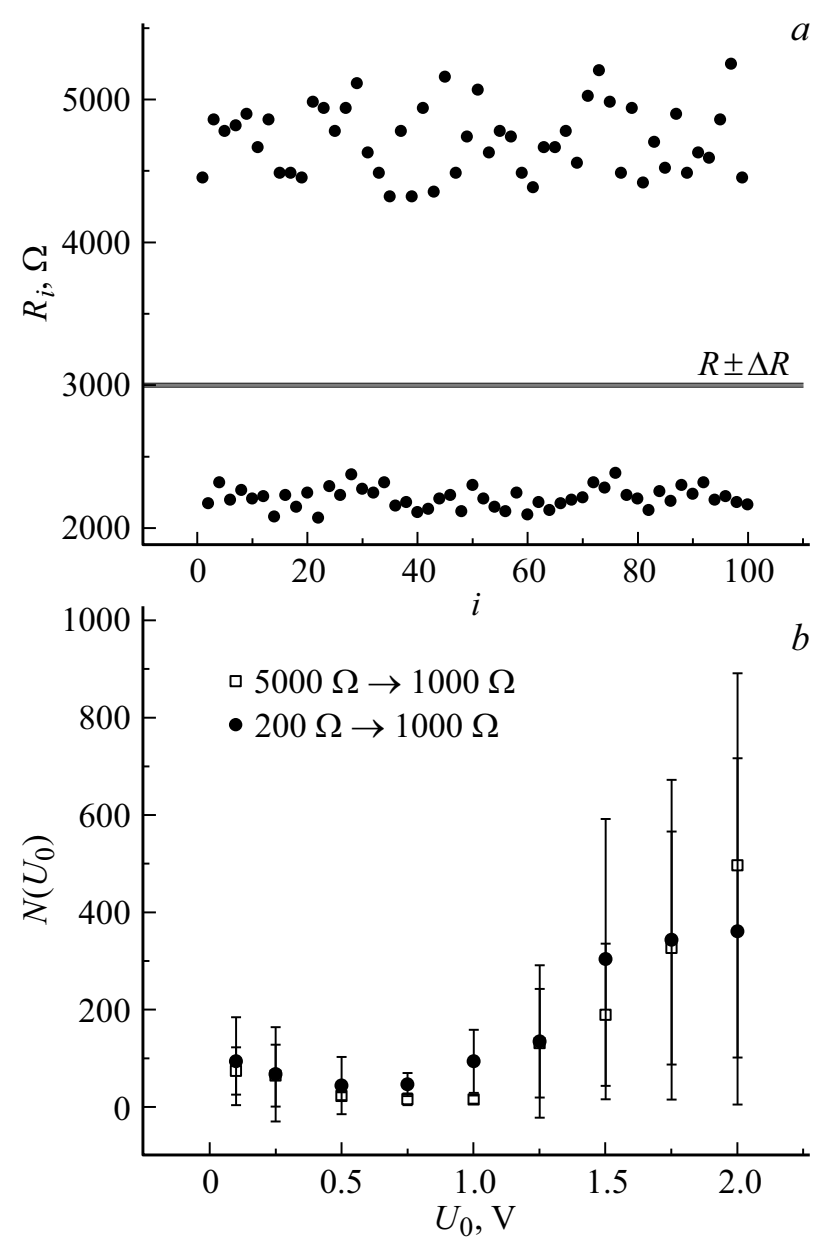

Рис. 2. $a-$ демонстрация проблемы сходимости алгоритма [10] при переходе из состояния с $R \sim 4700 \Omega$ в заданное состояние с $R=3000 \Omega$. При амплитуде $U_{0}=0.75 \mathrm{~V}$ происходит переход из состояния с $R \sim 4700 \Omega$ в состояние с $R \sim 2200 \Omega$ и обратно, состояние с $R=3000 \Omega$ недостижимо. $b$ - зависимость среднего количества итераций алгоритма $N$ от начальной амплитуды $U_{0}$ для процессов перехода из состояния с сопротивлением $5000 \Omega$ (квадраты) и $200 \Omega$ (кружки) в состояние с заданным сопротивлением $1000 \Omega$ для разработанной версии алгоритма. Статистика проводилась по десяти измерениям. 
сбрасывается до значения $U_{0}$. При изменении полярности более трех раз длительность обучающих импульсов начинает случайным образом изменяться в диапазоне от $0.9 \tau$ до $1.1 \tau$. Работа алгоритма завершается, когда текущее сопротивление структуры попадает в пределы заданной точности $\left(R-\Delta R<R_{i}<R+\Delta R\right)$.

На первом этапе была проведена апробация работоспособности алгоритма [11]. При этом было обнаружено, что в работе алгоритма при начальных импульсах большой амплитуды $U_{0}$ итерационный процесс перевода мемристора в состояние с заданным сопротивлением может не сходиться (происходит „зацикливание“): при подаче поочередно положительных и отрицательных импульсов текущее сопротивление на $i$-м шаге с хорошей точностью повторяет сопротивление на $(i-2)$-м шаге (рис. 2,a). Для того чтобы зацикливания не возникало, необходимо выбрать небольшое значение $U_{0}$ таким образом, чтобы импульс с данной амплитудой изменял сопротивление не более чем на $2 \Delta R$. Однако при выборе небольшого значения $U_{0}$ наблюдается резкое увеличение количества итераций для выхода на заданное значение сопротивления. Таким образом, время и стабильность работы алгоритма сильно зависят от начальной амплитуды $U_{0}$.

Для решения указанных проблем, как отмечалось выше, предлагается случайным образом в пределах 10\% изменять один из параметров алгоритма, например длительность импульсов. Длительность импульсов напряжения $(\tau=100 \mathrm{~ms})$ после трех изменений полярности начинает варьироваться в диапазоне $\pm 10 \%$ от начальной величины $\tau$. При таком изменении работа алгоритма становится более стабильной, зацикливания не наблюдается. С учетом этого дополнения был проведен подбор оптимальной начальной амплитуды импульсов $U_{0}$. На рис. $2, b$ изображена зависимость количества итераций от начальной амплитуды $U_{0}$ для переключения мемристорной структуры из состояний 200 и $5000 \Omega$ в $1000 \Omega$. Из рисунка видно, что для обоих направлений переключения существует некоторый диапазон начальных амплитуд, при которых наблюдается минимальное количество итераций, необходимых для перевода структуры $M / \mathrm{HK} / M$ в заданное резистивное состояние. Такие значения начальных амплитуд должны быть не слишком малыми, чтобы не увеличить количество итераций за счет малых изменений сопротивления $\left|R_{i}-R_{i-1}\right|$ на начальном этапе работы алгоритма, и не слишком большими, чтобы избежать зацикливания. Количество

Письма в ЖТФ, 2018, том 44, вып. 10 

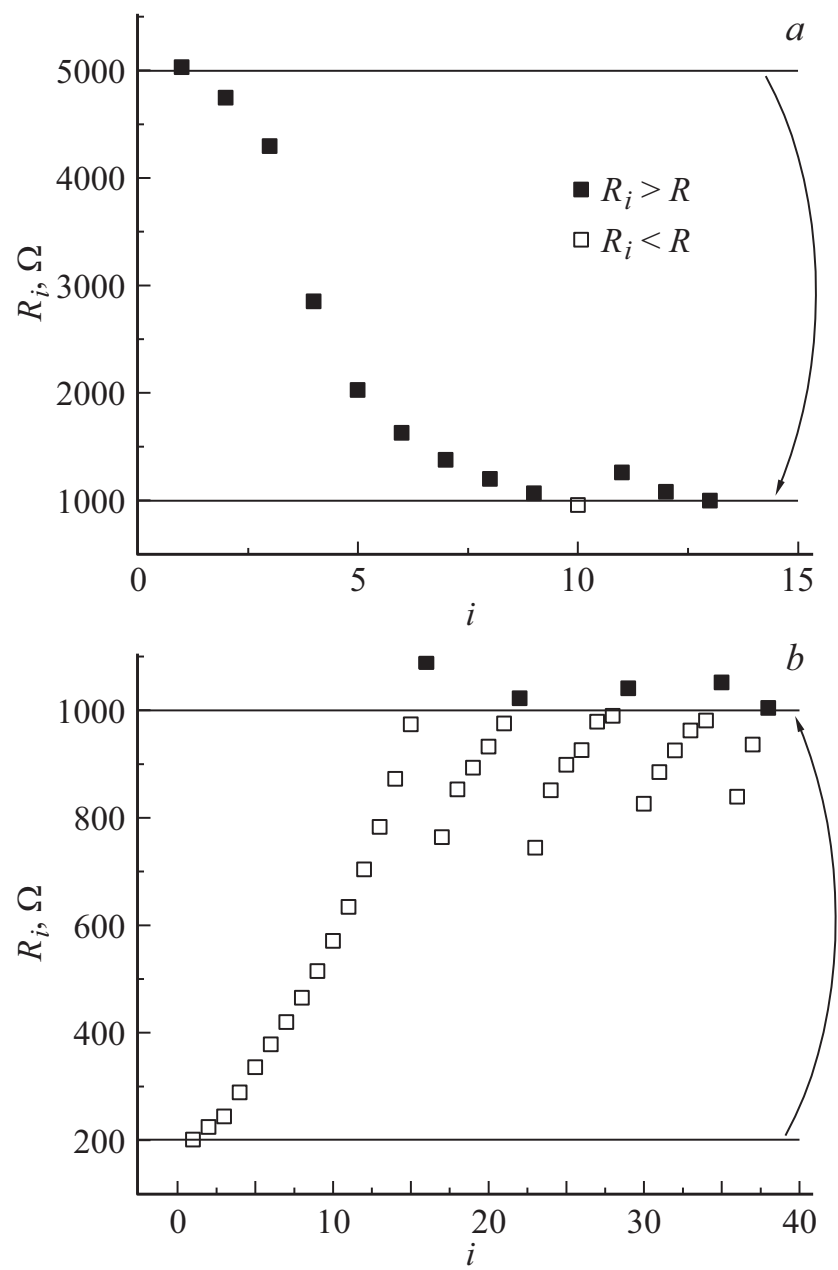

Рис. 3. Зависимость сопротивления $R_{i}$ мемристора от номера итерации алгоритма $i$. Показаны процессы переключения из состояния с сопротивлением $5000 \Omega$ в состояние с сопротивлением $1000 \Omega(a)$ и из состояния $200 \Omega$ в состояние $1000 \Omega(b)$. Амплитуда начального импульса $U_{0}=0.75 \mathrm{~V}$. Темными квадратами отмечены состояния $R_{i}>R$, при которых на $i$-й итерации подается положительный импульс, светлыми квадратами - состояния $R_{i}<R$, при которых на $i$-й итерации подается отрицательный импульс.

Письма в ЖТФ, 2018, том 44, вып. 10 
итераций алгоритма и соответственно время его работы минимальны при начальной амплитуде $U_{0}=0.5-1 \mathrm{~V}$.

Для начальной амплитуды $U_{0}=0.75 \mathrm{~V}$ был продемонстрирован процесс работы предложенной нами версии алгоритма. На рис. 3 изображен процесс перехода структуры $M / \mathrm{HK} / M$ из одного резистивного состояния в другое с точностью $0.5 \%$ : из 5000 в $1000 \Omega(a)$ и из 200 в $1000 \Omega(b)$. В первом случае (рис. 3,a) алгоритм успешно завершает работу после двух изменений полярности импульсов напряжения. Однако во втором варианте (рис. $3, b$ ) только изменения полярности и амплитуды импульсов уже недостаточно для успешного завершения работы алгоритма, при котором не возникает зацикливание, показанное на рис. 2, $a$. В этом случае влияние случайных изменений длительности импульсов напряжения оказывается существенным для перехода структуры $M / \mathrm{HK} / M$ в заданное состояние с $R=1000 \Omega$.

Таким образом, в работе был реализован алгоритм перевода мемристорной структуры в заданное резистивное состояние с точностью не хуже $0.5 \%$ в условиях, когда такой переход затруднен, только путем изменения полярности и амплитуды импульсов напряжения, воздействующих на структуру. Алгоритм основан на подаче импульсов напряжения с плавно растущей амплитудой и случайным образом изменяющейся длительностью. Подобраны оптимальные параметры для работы алгоритма с минимальным количеством итераций.

Работа выполнена при финансовой поддержке Российского научного фонда (грант № 16-19-10233) на оборудовании Ресурсного центра электрофизических методов (НИЦ „Курчатовский институт“).

\section{Список литературы}

[1] Strukov D.B., Snider G.S., Stewart D.R., Williams R.S. // Nature. 2008 . V. 453. P. 80-83.

[2] Lee J.S., Lee S., Noh T.W. // Appl. Phys. Rev. 2015. V. 2. P. 031303.

[3] Ielmini D. // Semicond. Sci. Technol. 2016. V. 31. P. 063002.

[4] Emelyanov A.V., Lapkin D.A., Demin V.A., Erokhin V. V., Battistoni S., Baldi G., Dimonte A., Korovin A.N., Iannotta S., Kashkarov P.K., Kovalchuk M.V. // AIP Advances. 2016. V. 6. P. 111301.

[5] Demin V.A., Emelyanov A.V., Lapkin D.A., Erokhin V.V., Kashkarov P.K., Kovalchuk M.V. // Cryst. Rep. 2016. V. 61. N 6. P. 992-1001.

Письма в ЖТФ, 2018, том 44, вып. 10 
[6] Alibart F., Zamanidoost E., Strukov D.B. // Nature Commun. 2013. V. 4. P. $2072(1-7)$.

[7] Jo K.-H., Jung C.-M., Min K.-S., Kang S.-M. // IEEE Trans. Nanotechnol. 2010. V. 9. N 6. P. 675-678.

[8] Kim K.M., Lee S.R., Kim S., Chang M., Hwang C.S. // Adv. Funct. Mater. 2015. V. 25. N 10. P. $1527-1534$.

[9] Vourkas I., Gómez J., Abusleme Á., Vasileiadis N., Sirakoulis G.Ch., Rubio A. Exploring the voltage divider approach for accurate memristor state tuning // LASCAS-2017. Bariloche, 2017. P. 1-4.

[10] Kim K.M., Yang J.J., Strachan J.P., Grafals E.M., Ge N., Melendez N.D., Li Z., Williams R.S. // Sci. Rep. 2016. V. 6. P. 20085.

[11] Alibart F., Gao L., Hoskins B.D., Strukov D.B. // Nanotechnology. 2012. V. 23. P. 075201.

[12] Калинин Ю.Е., Ремизов А.Н., Ситников А.В. // ФТТ. 2004. Т. 46. В. 11. C. 2076-2082.

[13] Рыльков В.В., Николаев С.Н., Демин В.А., Емельянов А.В., Ситников А.В., Никируй К.Э., Леванов В.А., Пресняков М.Ю., Талденков А.Н., Васильев А.Л., Черноглазов К.Ю., Веденеев А.С., Калинин Ю.Е., Грановский А.Б., Тугушев В.В., Бугаев А.С. // ЖЭТФ 2018. Т. 153. В. 3. С. 424-441.

[14] Udalov O.G., Chtchelkatchev N.M., Glatz A., Beloborodov I.S. // Phys. Rev. B. 2014. V. 89. P. 054203.

[15] Shaposhnikov A.V., Perevalov T.V., Gritsenko V.A., Cheng C.H., Chin A. // Appl. Phys. Lett. 2012. V. 100. P. 243506. 